\title{
Analysing velocity data for emergency cars at urban crossroads
}

\author{
M. Woelki, R. Nippold \& S. Ruppe \\ Institute of Transportation Systems, \\ German Aerospace Center (DLR), Germany
}

\begin{abstract}
Concerning disaster management with respect to urban transport, one vital and lifesaving part of this sector is the driving of emergency cars. In urban German areas, firemen have to reach their destination within a legal time of eight minutes within $90 \%$ of the cases of emergency. In order to achieve that, they are equipped with special right-of-way when activating their flashing blue light. They can drive with increased speed, they have priority, they can use emergency lanes formed by other cars and they can pass over red traffic lights. However, although they are equipped with these special rights, they have to be careful that no accidents happen and that nobody is injured. Consequently, at each intersection they typically have to reduce their speed. Therefore, it is difficult to predict for a given fire station the actual region that can be reached within the legal time. Obviously, crossings form a major bottleneck along their route. Therefore, the present paper considers real data for emergency cars at crossroads. The velocity data of emergency cars passing the crossroad are compared in case of driving (1) with blue-flashing light and siren, (2) only blue-light driving, and (3) without any signal. The results are a first step towards a realistic prediction of the trajectories of emergency cars in case of emergency and help to identify bottlenecks that should be avoided if possible. The results can be used for special routing for emergency cars and are of importance for typical cases of emergency as well as large-scale applications of disaster management.
\end{abstract}

Keywords: urban transport, routing, GPS-data, velocity profile, Brownian Bridge. 


\section{Introduction}

In the city of Brunswick the local fire department operates a modern, computeraided dispatching system by the German enterprise Convexis $\mathrm{GmbH}$ [1]. Their system RescueTrack $^{\circledR}$ [2] tracks the current positions of emergency vehicles in order to assign missions to the emergency vehicle with shortest distance to the place of action. As part of a research project, the German Aerospace Center (DLR) receives data from several emergency vehicles. The data set used in this paper comprises data from more than one year and includes a time-stamp, the current geo position and information about the usage of blue-light and horn signals in a temporal resolution of five seconds. The attention is focused on three intersections comprising around 43000 GPS measurement points. The research project is called VABENE++ [3] and deals mainly with traffic management for large scale events and disasters. With the help of its traffic information system EmerT [4] developed for data exchange between traffic management centers and emergency services the operational control can select an appropriate rescue team with minimal travel time to the operating site. The system indicates the current travel time for the arrival of the respective team. Moreover, after alerting, EmerT presents the officer of the receptive team a route recommendation which is adapted for the current emergency situation and coordinated with the operational control. An up-to-date position of the rescue team is continuously displayed on the digital map. Additionally, an expected arrival time is reported the operational control. Figure 1 shows two exemplary missions of emergency vehicles to the place of operation and afterwards to a hospital.

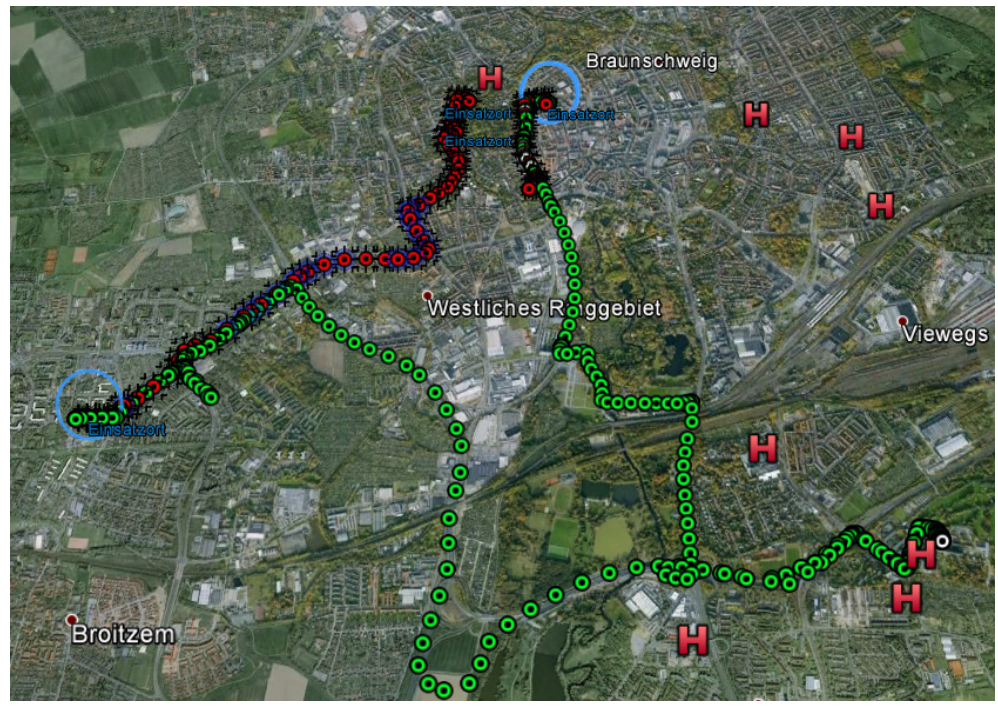

Figure 1: Two missions of emergency cars. Blue circles show the place of operation while the hospitals are indicated by $\mathrm{H}$. The map is taken from Google Earth [5]. 
The present paper aims to analyse how the crossing of urban intersections retards emergency vehicles despite of their special right-of-way. Previous investigations in VABENE++ provided EmerT with a special routing for emergency cars [6] and an analysis of strategically optimal routes with regard to dynamic alternatives [7]. Therewith the present investigation complements the existing research in order to describe emergency traffic most realistically by taking into account detailed velocity data depending on the state of the emergency cars. This will in a later stage also affect the routing in case of emergency.

Typical velocity profiles for a whole trip that were measured along with a smoothing spline are given in Figure 2.

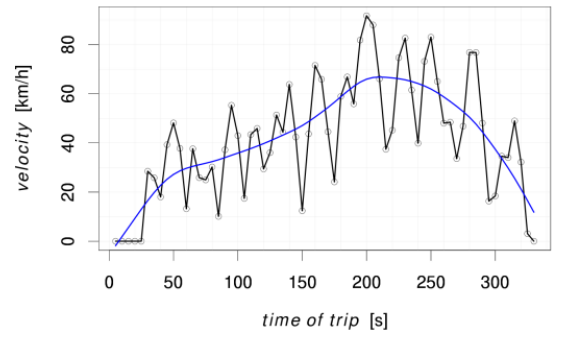

(a)

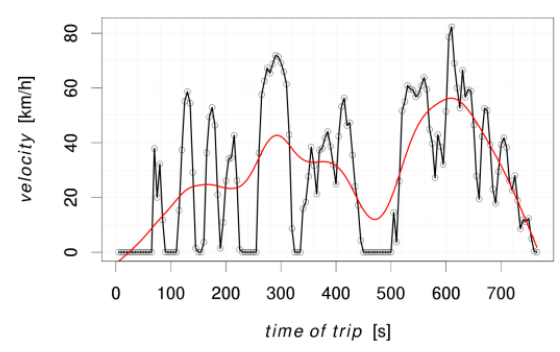

(b)

Figure 2: Two measured velocity profiles corresponding to Figure 1. (a) The car drives from the fire station to the destination and uses blue-light and horn. (b) The car drives with the patient to the hospital (without any signal).

\section{Algorithm for data extraction}

To analyze the crossing of intersections, the areas of the intersections under consideration need to be extracted from the collected data set. For this reason, we have defined an investigation area for each intersection. Only data of those areas has been taken into consideration. A procedure was invented that matches the GPS data to reference points, whereby each reference point represents an entrance to the intersection. The reference points are obtained as the midpoint of clusters of start- and endpoints of each trajectory in this intersection area. After the reference points were calculated, each start- and endpoints of trajectories is matched to the closest one of them. Finally, for each investigation area the trajectories along with the turning direction are available.

\section{Measured speeds}

This section analyses the measured velocities from ambulance vehicles recorded between 29 October 2013 and 18 December 2014 in the city of Brunswick, 
Germany. The analysis concentrates on three comparable intersections of federal roads in the inner-city area with consistent high traffic volume.

In a first step, the daily velocity profile of the ambulance vehicles in different modes of operation was analyzed. Figure 3 shows the measured GSP velocities (black points) and smoothed daily courses for normal trips without any special signals used (black line), with blue flashing lights activated (blue line) and with additional siren in operation (red line). Trips without any special signals used are equal to normal vehicular traffic. Trips with special signals always indicate rescue operations and therefore show considerable higher speeds.

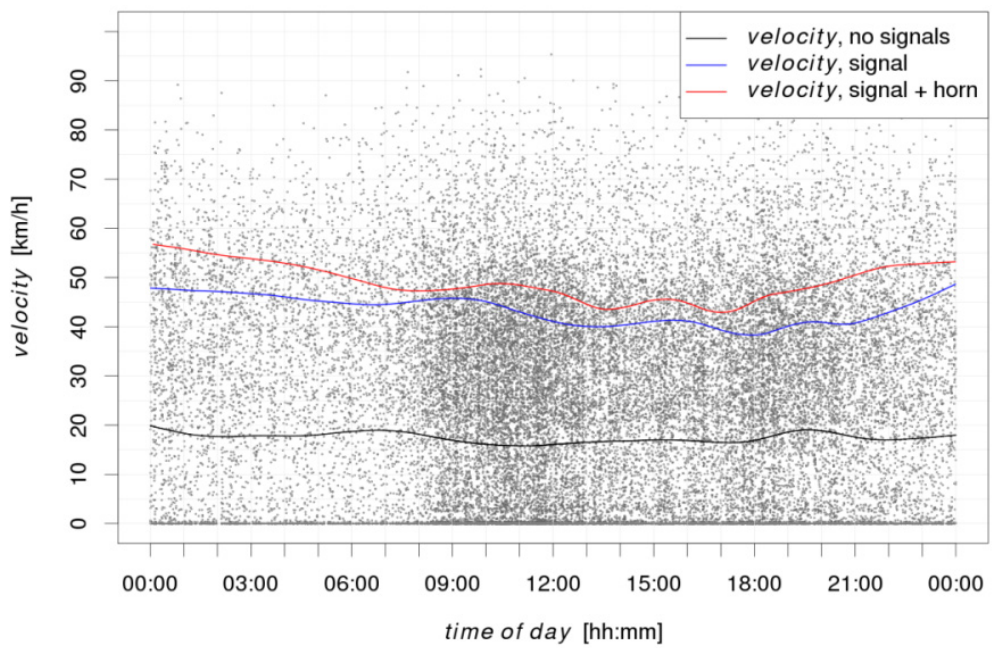

Figure 3: Daily course of velocity for emergency vehicles at three inner-city intersections of federal roads.

There are large differences between the velocities for trips without any special signals and trips with signals active. In contrast, variations between trips with only blue flashing lights and trips with blue flashing lights and sirens used are rather small. This observation is supported by the fact that sirens are only active in case of obstacles, e.g. other cars in front of red traffic light or blockages caused by a missing rescue lane.

Figure 4 looks more into detail. The bars in the background indicate the frequencies of the measured GPS-velocities. The solid lines in the foreground show the resulting density curve. Again, the black colour indicates trips without any signals used. Blue and red colours describe trips with only blue flashing lights used and trips with blue flashing lights and sirens activated.

Here, the distribution of the velocities occurring for different modes of operation of ambulance vehicle clearly shows differences between trips without any special signals and tips with signal activated. The black lines for trips without any signal has a high peak at $v=0 \mathrm{~km} / \mathrm{h}$ (stand-still). The bar for $v=0 \mathrm{~km} / \mathrm{h}$ was even cut off because of its high magnitude in comparison to other occurring velocities. This addresses the fact of waiting vehicles in front of 
a red traffic light. Note in addition the steeper slope of the black curve for velocities greater than $45 \mathrm{~km} / \mathrm{h}$. The blue curve for trips with blue flashing lights activated shows very low frequencies for velocities below $20 \mathrm{~km} / \mathrm{h}$ and a peak at $v=35 \mathrm{~km} / \mathrm{h}$. The red curve for trips with the highest priority using siren in addition reaches its peak at $v=60 \mathrm{~km} / \mathrm{h}$. Together with the blue curve it shows a less steep drop for velocities greater than $45 \mathrm{~km} / \mathrm{h}$ is in comparison to the black curve.

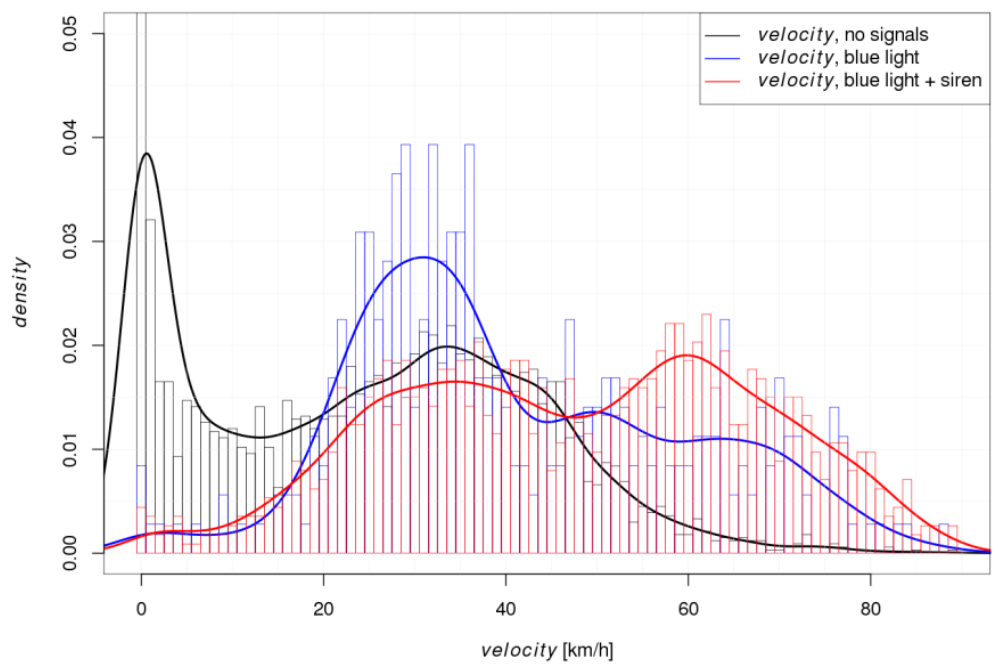

Figure 4: Histogram of velocities for emergency vehicles at three inner-city intersections of federal roads.

Figure 5 analyses the driving process at the intersection in greater detail. Here, the deceleration and acceleration means are represented by the recoded GPS trajectory data. These trajectories are disposed in the way that the time step with the lowest velocity or the last time step at stand-still (velocity equals zero) lays at 0 seconds. This should meet the stop line of an intersection since interferences with other vehicles are not likely to occur behind the intersection.

Acceleration is indicated by the gradient of the lines, velocity at the intersection by the intercept. The black colour stands for trips without any signals used. Blue and red colours describe trips with only blue flashing lights used and trips with blue flashing lights and sirens activated. The thin lines in the background indicate the single trajectories of the emergency vehicles for approaching and leaving the intersection. The thick lines symbolise a linear fit of all single trajectories.

Trips without any special signals (grey thin lines resp. the black thick line) are comparable to normal vehicular traffic and therefore show a certain amount of stand-still in front of intersections. This fact is represented by a number of almost horizontal trajectories in the lower left corner of the plot. In result, a significant lower velocity (lower intercept) and acceleration (less steep gradient) is indicated by the black line. 


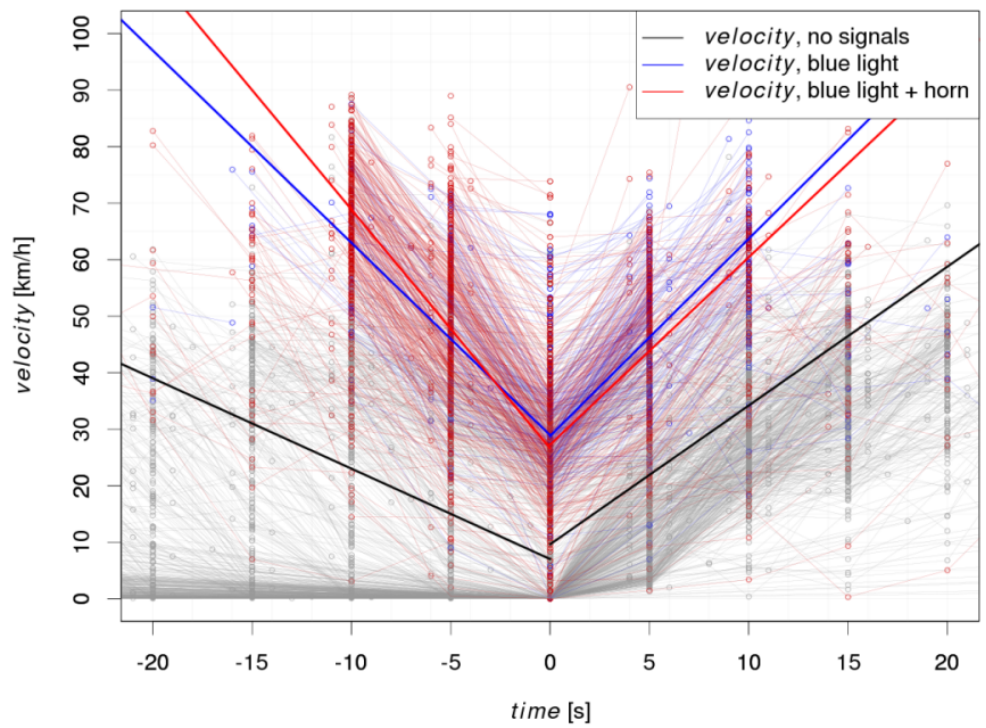

Figure 5: Graphical representation of the deceleration and acceleration processes at three inner-city intersections of federal roads.

In accordance with Figure 3, only small variations occur between trips with blue flashing lights and trips with blue flashing lights and siren used. The following Table 1 comprises the underlying values for the graphical representation in Figure 5.

Table 1: Analytical representation of the deceleration and acceleration processes for different mode of operation at three inner-city intersections of federal roads.

\begin{tabular}{|l|c|c|c|c|}
\hline \multirow{2}{*}{$\begin{array}{l}\text { Mode of } \\
\text { operation }\end{array}$} & \multicolumn{2}{|c|}{ Approaching } & \multicolumn{2}{c|}{ Leaving } \\
\cline { 2 - 5 } & $\begin{array}{c}\text { Gradient } \\
{\left[\mathrm{m} / \mathrm{s}^{2}\right]}\end{array}$ & $\begin{array}{c}\text { Intercept } \\
{[\mathrm{km} / \mathrm{h}]}\end{array}$ & $\begin{array}{c}\text { Gradient } \\
{\left[\mathrm{m} / \mathrm{s}^{2}\right]}\end{array}$ & $\begin{array}{c}\text { Intercept } \\
{[\mathrm{km} / \mathrm{h}]}\end{array}$ \\
\hline No signals & -0.44 & 7.01 & 0.68 & 9.62 \\
\hline $\begin{array}{l}\text { Blue flashing } \\
\text { lights }\end{array}$ & -0.95 & 28.93 & 0.97 & 28.79 \\
\hline $\begin{array}{l}\text { Blue flashing } \\
\text { lights + siren }\end{array}$ & -1.17 & 26.59 & 0.92 & 27.27 \\
\hline
\end{tabular}

In a second step, the daily velocity profile of the ambulance vehicles for different directions was analyzed. Figure 6 shows the measured GSP velocities (black points) and smoothed daily courses for trips passing the intersection straight ahead. The blue line indicates right turning trips and the red line trips with left turns at the intersections. 


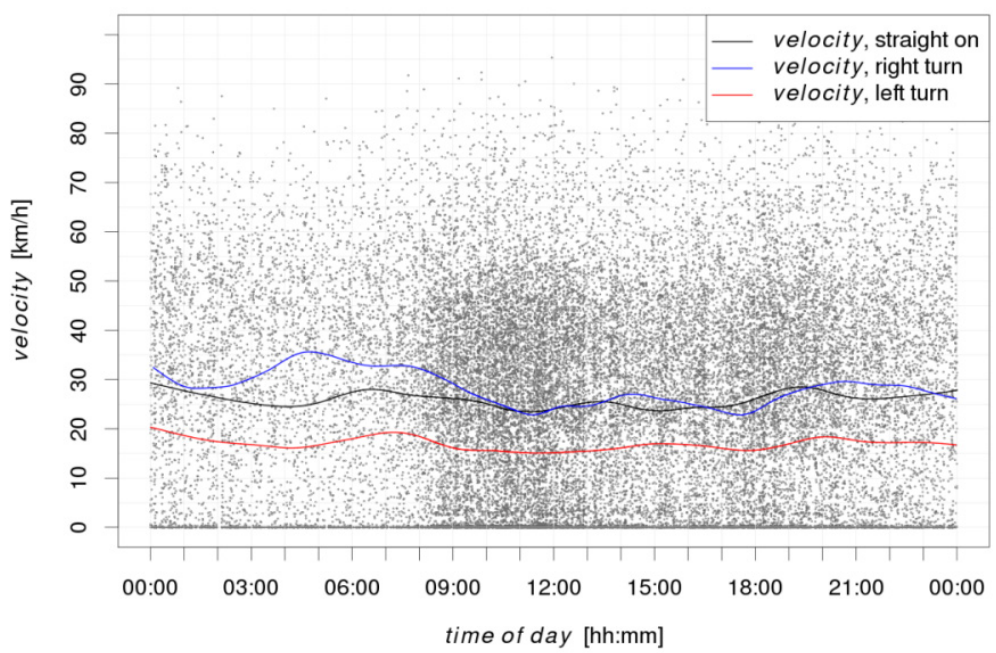

Figure 6: Daily course of velocity for emergency vehicles at three inner-city intersections of federal roads.

The analysis shows rather small variations between trips with right turns and trips passing the intersection straight ahead. However, right turn trips reveal a slightly greater velocity than trips leading straight on. This is explained by the fact that for the case of right turns, only vehicles coming from left as well as pedestrians crossing the street have to be considered. Nevertheless, even greater velocities are not possible especially during patient transfer.

Trips passing the intersection straight ahead need to consider vehicular traffic from left and right and again pedestrians crossing the street. Trips turning left need to consider vehicular traffic from left, right and ahead in the opposite direction and additionally pedestrians. Therefore, they have a considerable lower velocity indicated in Figure 6. The histograms of velocities, however, show only little differences between the direction of trips and are not depicted here.

Figure 7 again analyses the deceleration and acceleration processes in greater detail. In accordance with Figure 6 the black line indicates the mean velocity of all trips going straight ahead over the intersection. The blue line represents trips with right turns at the intersection whereas left turns are characterized by the red line. The usage of signal was not considered.

Here, the recoded GPS trajectory data represent the directions of the trips. The trajectories themselves are disposed in the same way as for Figure 5.

The analysis shows almost the same deceleration and acceleration rates (gradients of the thick lines) for all directions. In accordance with Figure 4, however, there is a significant lower velocity for trips turning left. This behaviour is also represented by the calculated values for the mean velocity at the intersection (intercept) and the mean deceleration and acceleration (gradient) in Table 2. 


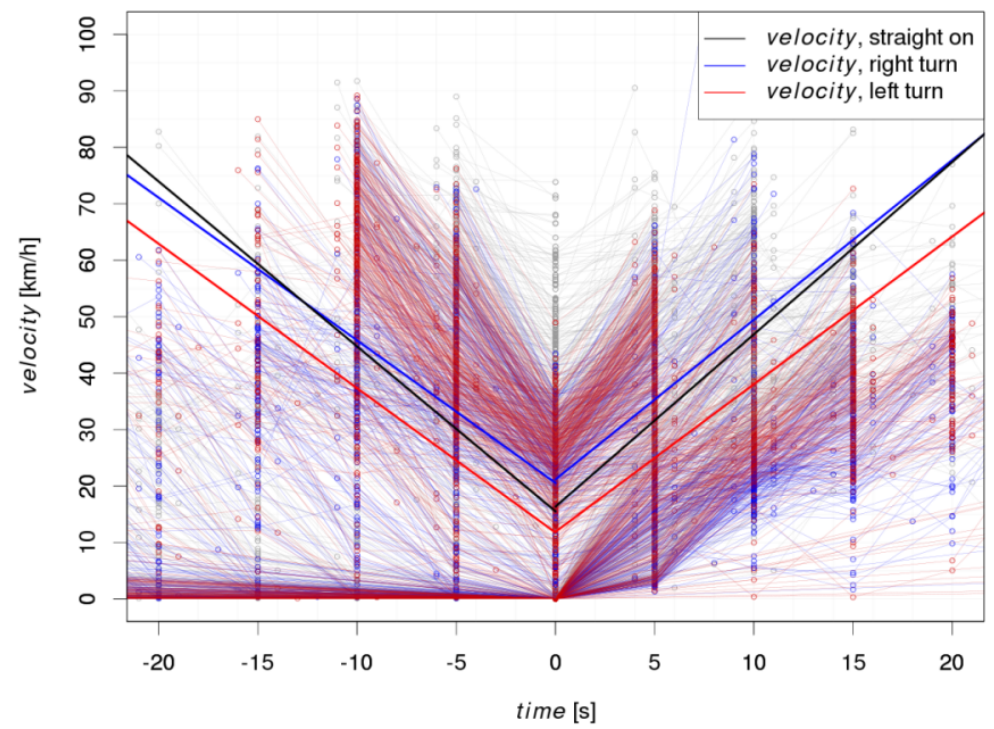

Figure 7: Graphical representation of deceleration and acceleration processes at three inner-city intersections of federal roads.

Table 2: Analytical representation of the deceleration and acceleration processes for different direction of trips at three inner-city intersections of federal roads.

\begin{tabular}{|l|c|c|c|c|}
\hline \multirow{2}{*}{ Direction of trip } & \multicolumn{2}{|c|}{ Approaching } & \multicolumn{2}{c|}{ Leaving } \\
\cline { 2 - 5 } & $\begin{array}{c}\text { Gradient } \\
{\left[\mathbf{m} / \mathbf{s}^{2}\right]}\end{array}$ & $\begin{array}{c}\text { Intercept } \\
{[\mathbf{k m} / \mathbf{h}]}\end{array}$ & $\begin{array}{c}\text { Gradient } \\
{\left[\mathbf{m} / \mathbf{s}^{2}\right]}\end{array}$ & $\begin{array}{c}\text { Intercept } \\
{[\mathbf{k m} / \mathbf{h}]}\end{array}$ \\
\hline Straight on & -0.81 & 15.50 & 0.85 & 16.34 \\
\hline Right turn & -0.70 & 20.53 & 0.79 & 21.16 \\
\hline Left Turn & -0.71 & 11.74 & 0.73 & 11.88 \\
\hline
\end{tabular}

In Figure 8 one sees that while driving with blue-light and horn the emergency cars reach mean speeds between forty and fifty kilometres per hour with a variance of around $20 \mathrm{~km} / \mathrm{h}$. The driving direction has rather less influence on the speed distribution. Velocities near zero appear very rarely. Concluding, one can state that emergency cars in Brunswick can make very effective use of their special rights-of-way. The velocity distribution changes dramatically in the right diagram. Here one sees that the speed average (along with the median) sinks below $20 \mathrm{~km} / \mathrm{h}$. While with blue-light and horn left turning is performed with more than $40 \mathrm{~km} / \mathrm{h}$ on average (in the considered intersection area), without blue-light this value tends to zero. 

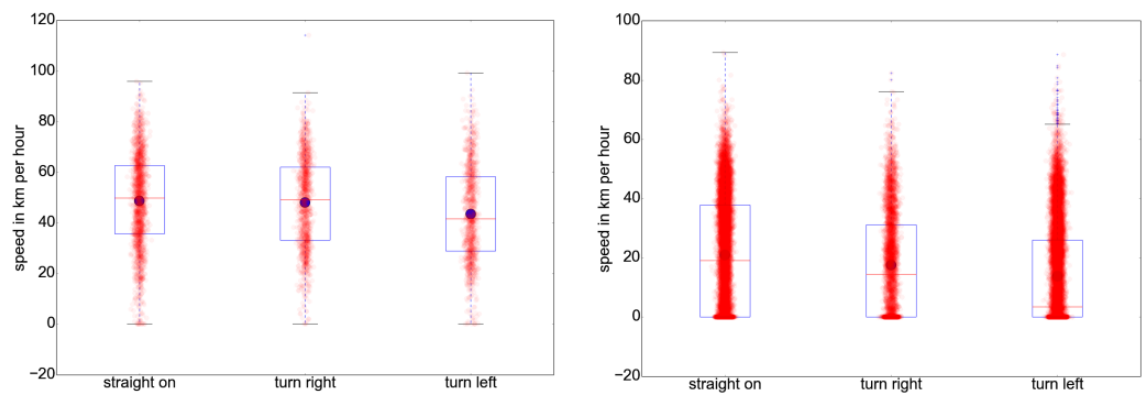

Figure 8: Mean speed distributions averaged over three comparable intersections and daily times. The blue dots represent the mean, the blue boxes the variance and the red horizontal lines the median.

\section{Summary and outlook}

This paper investigated velocity data from emergency vehicles in Brunswick with regard to their mode of operation that mainly differs by either using special rights of way (blue flashing light with or without siren) and by driving homogeneously with the traffic flow of the background vehicles (without any signal). Examples of typical velocity profiles for a whole mission showed that driving with special rights of way typically leads to a continuously moving state without in-between stops while driving without signal is strongly dominated by stop and go. The main part of the paper focused on the velocity data in the vicinity of urban crossings. Three comparable intersections of equally high capacity and constantly high traffic demand have been analyzed. However, investigations of other crossings showed typically the same behaviour on average.

The average decelerations and accelerations as well as the velocities in the daily course have been extracted. One also observes slight differences for working days and weekends, as shown in Figure 9.

The velocity profiles of emergency cars in certain modes of operation can be modelled by an appropriate Gaussian process [8]. The idea is to consider a Brownian-Bridge [9] that is a stochastic process on the interval $t \in[0,1]$ with zero mean and variance $t(1-t)$. It has the conditional probability distribution of a Wiener process $W(t)$ and is conditioned to start and end on the x-axis: $B(0)=B(1)=0$. The relation to the standard Wiener process can be written as $B(t)=W(t)-t W(1)$. This is easily programmed with the help of the midpoint-displacement method $[10,11]$.

Typically a Brownian Bridge can take on positive and negative values. However, since in the present application we consider velocities we restrict the Bridge to the first quadrant. In the left diagram of Figure 2 the car drives with flashing light and siren and is therewith in a state where the velocity will not vanish apart from the start and the end. This leads to a so-called Brownian Excursion in which the mean of the Gaussian is increased to positive velocities. To reproduce the halting times of the right-hand diagram we run a usual 


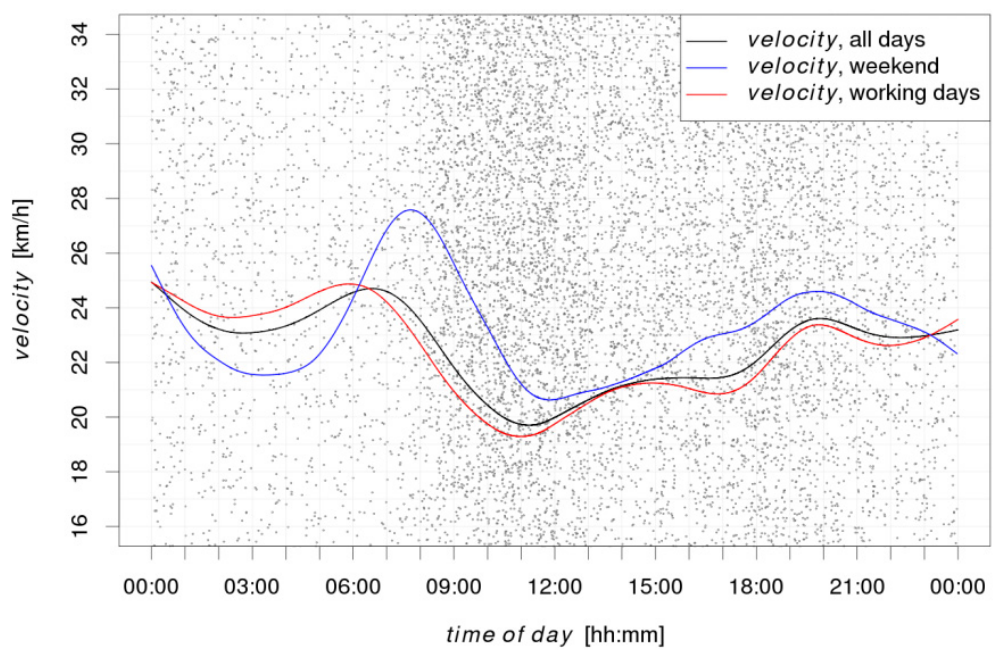

Figure 9: Daily course of velocity for emergency vehicles at three inner-city intersections of federal roads.
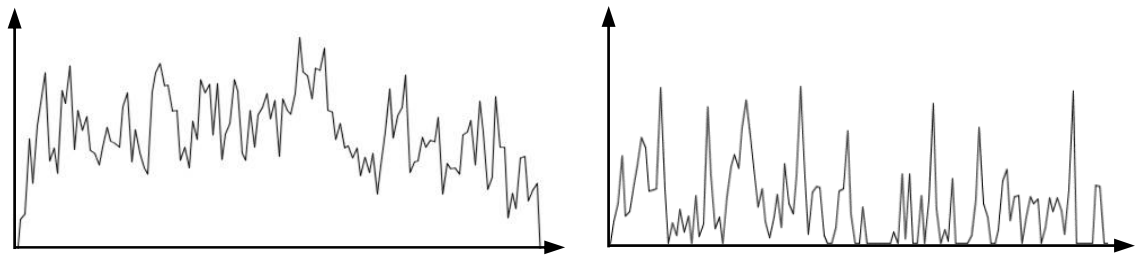

Figure 10: $\quad$ Modified Brownian-Bridges modelling the profiles of Figure 2.

Brownian-Bridge process and cancel all negative velocities: $v \rightarrow \max (v, 0)$. This leads to the right diagram of Figure 2.

The Brownian Bridge shall be parametrized by the measured data. A detailed analysis of whole velocity profiles will be published elsewhere.

\section{Acknowledgements}

The authors would like to thank Benjamin Jauerka and Günter Kuhns for providing the data. The work was part of the VABENE++ project [3]. 


\section{References}

[1] Convexis GmbH Gerhard-Kindler-Straße 372770 Reutlingen info@convexis.de www.convexis.de

[2] Software RescueTrack ${ }^{\circledR}$ : www.rescuetrack.de

[3] http://www.dlr.de/vabene/en/desktopdefault.aspx

[4] http://www.dlr.de/vabene/en/desktopdefault.aspx/tabid-9048/15657_read39143/

[5] earth.google.de, 2015-01-14

[6] Woelki, M., Nippold, R., Bonert, M., and Ruppe, S. Risk-minimal routes for emergency cars. In: Disaster Management and human health risk III, 159-169. WIT Press. 09.-11. Juli 2013, A Coruna, Spanien. WIT, UK. ISBN 978-1-84564-738-4. ISSN 1746-4498 (2013)

[7] Woelki, M., Lu, T., and Ruppe, S.: Ranking of Alternatives for Emergency Routing on Urban Road Networks. Acc. f. publ. in: Urban Transport 2015. Urban Transport 2015, 02.-04. June 2015, Spain. WIT, UK (2015)

[8] MacKay, D. J. C. Introduction to Gaussian processes. In C.M. Bishop, editor, Neural Networks and Machine Learning, volume 168 of NATO ASI Series, pages 133-165. Springer, Berlin, 1998

[9] Revuz, D.; Yor, M. Continuous Martingales and Brownian Motion (2nd ed.). New York: Springer-Verlag. ISBN 3-540-57622-3_(1999)

[10] Lau, W. C., Eramilli, A., Wang, J. L., Willinger, W.: Self-similar traffic generation: the random midpoint displacement algorithm and its properties. In: Proc. ICC'95, Seattle, pp.446-472 (1995)

[11] http://introcs.cs.princeton.edu/java/23recursion/Brownian.java, 2015-0114 